\title{
Transformations of polar Grassmannians preserving certain intersecting relations
}

\author{
Wen Liu • Mark Pankov • Kaishun Wang
}

Received: 8 July 2013 / Accepted: 8 January 2014 / Published online: 15 January 2014

(C) Springer Science+Business Media New York 2014

\begin{abstract}
Let $\Pi$ be a polar space of rank $n \geq 3$. Denote by $\mathcal{G}_{k}(\Pi)$ the polar Grassmannian formed by singular subspaces of $\Pi$ whose projective dimension is equal to $k$. Suppose that $k$ is an integer not greater than $n-2$ and consider the relation $\mathfrak{R}_{i, j}, 0 \leq i \leq j \leq k+1$, formed by all pairs $(X, Y) \in \mathcal{G}_{k}(\Pi) \times \mathcal{G}_{k}(\Pi)$ such that $\operatorname{dim}_{p}\left(X^{\perp} \cap Y\right)=k-i$ and $\operatorname{dim}_{p}(X \cap Y)=k-j\left(X^{\perp}\right.$ consists of all points of $\Pi$ collinear to every point of $X)$. We show that every bijective transformation of $\mathcal{G}_{k}(\Pi)$ preserving $\Re_{1,1}$ is induced by an automorphism of $\Pi$, except the case where $\Pi$ is a polar space of type $\mathrm{D}_{n}$ with lines containing precisely three points. If $k=n-t-1$, where $t$ is an integer satisfying $n \geq 2 t \geq 4$, we show that every bijective transformation of $\mathcal{G}_{k}(\Pi)$ preserving $\Re_{0, t}$ is induced by an automorphism of $\Pi$.
\end{abstract}

Keywords Polar space · Polar Grassmannian · Intersecting relation · Automorphism

W. Liu $\cdot$ K. Wang $(\varangle)$

Sch. Math. Sci. \& Lab. Math. Com. Sys., Beijing Normal University, Beijing, 100875, China e-mail: wangks@bnu.edu.cn

W. Liu

e-mail: liuwen1975@126.com

W. Liu

Hebei Key Lab of Computational Mathematics \& Applications, and College of Math \& Info.

Sciences, Hebei Normal University, Shijiazhuang 050024, China

M. Pankov

Department of Mathematics and Computer Science, University of Warmia and Mazury, Olsztyn, Poland

e-mail: pankov@matman.uwm.edu.pl 


\section{Introduction}

Let $V$ be an $n$-dimensional vector space $V$ (over a division ring). Denote by $\mathcal{G}_{k}(V)$ the Grassmannian formed by the $k$-dimensional subspaces of $V$. Suppose that $1<$ $k \leq n-k$. For every integer $i$ satisfying $1 \leq i \leq k$, we define the following relation:

$$
\mathfrak{R}_{i}:=\left\{(X, Y) \in \mathcal{G}_{k}(V) \times \mathcal{G}_{k}(V): \operatorname{dim}_{l}(X \cap Y)=k-i\right\} .
$$

We write $\operatorname{dim}_{l}$ for the linear dimension (the dimension of vector spaces and their subspaces) since we want to distinguish it from the projective dimension (the dimension of projective spaces and their subspaces), which will be denoted by $\operatorname{dim}_{p}$. Note that $(X, Y) \in \mathfrak{R}_{i}$ if and only if the distance between $X$ and $Y$ in the Grassmann graph

$$
\Gamma_{k}(V)=\left(\mathcal{G}_{k}(V), \Re_{1}\right)
$$

is equal to $i$.

The same relations can be defined on dual polar spaces. Let $\Pi$ be a polar space of rank $n$. Denote by $\mathcal{G}_{k}(\Pi)$ the polar Grassmannian formed by singular subspaces of $\Pi$ whose projective dimension is equal to $k$. The associated dual polar space is formed by maximal singular subspaces, i.e., singular subspaces of dimension $n-1$. For any integer $i$ satisfying $1 \leq i \leq n$, we define

$$
\mathfrak{R}_{i}:=\left\{(X, Y) \in \mathcal{G}_{n-1}(\Pi) \times \mathcal{G}_{n-1}(\Pi): \operatorname{dim}_{p}(X \cap Y)=n-1-i\right\} .
$$

As above, we have $(X, Y) \in \mathfrak{R}_{i}$ if and only if the distance between $X$ and $Y$ in the dual polar graph

$$
\Gamma_{n-1}(\Pi)=\left(\mathcal{G}_{n-1}(\Pi), \mathfrak{R}_{1}\right)
$$

is equal to $i$.

By [4], every automorphism of the Grassmann graph $\Gamma_{k}(V)$ is induced by a semilinear automorphism of $V$ or a semilinear isomorphism of $V$ to the dual vector space $V^{*}$ (the second possibility can be realized only in the case $n=2 k$ ). Similarly, every automorphism of the dual polar graph $\Gamma_{n-1}(\Pi)$ is induced by an automorphism of the polar space $\Pi$. The latter was proved by Chow [4] for classical polar spaces only, but Chow's method works in the general case [19, Sect. 4.6]. Some results closely related to these statements were obtained [7-12, 16, 20, 21], and we refer [19] for a survey.

Every bijective transformation of $\mathcal{G}_{k}(V)$ preserving $\mathfrak{R}_{k}$ is an automorphism of $\Gamma_{k}(V)[2,9]$. For the relation $\mathfrak{R}_{i}$ with $1<i<k$ the same is not proved. However, all bijective transformations of $\mathcal{G}_{k}(V)$ preserving $\Re_{1} \cup \cdots \cup \mathfrak{R}_{m}$ are automorphisms of $\Gamma_{k}(V)$ for every integer $m<k$ [17]. This is a generalization of the previous result; indeed, if $m=k-1$, then the transformations considered above preserve $\mathfrak{R}_{k}$. The same statement is proved for some dual polar spaces [14]. Results of similar nature were established for other objects $[1,5,6,13,15]$.

Now suppose that $k$ is an integer not greater than $n-2$ and consider the relation $\mathfrak{R}_{i, j}, 0 \leq i \leq j \leq k+1$, formed by all pairs

$$
(X, Y) \in \mathcal{G}_{k}(\Pi) \times \mathcal{G}_{k}(\Pi)
$$


satisfying the following conditions:

$$
\operatorname{dim}_{p}\left(X^{\perp} \cap Y\right)=k-i \quad \text { and } \quad \operatorname{dim}_{p}(X \cap Y)=k-j
$$

( $X^{\perp}$ consists of all points of $\Pi$ collinear to every point of $X$ ). All automorphisms of the polar Grassmann graph

$$
\Gamma_{k}(\Pi)=\left(\mathcal{G}_{k}(\Pi), \Re_{0,1}\right)
$$

are described in [19, Sect. 4.6]. They are induced by automorphisms of $\Pi$, except the case where $k=1$ and our polar space is of type $D_{4}$. Also, every automorphism of the so-called weak Grassmann graph

$$
\Gamma_{k}^{w}(\Pi)=\left(\mathcal{G}_{k}(\Pi), \Re_{0,1} \cup \Re_{1,1}\right)
$$

is induced by an automorphism of $\Pi$ [22].

We show that every bijective transformation of $\mathcal{G}_{k}(\Pi)$ preserving $\Re_{1,1}$ is induced by an automorphism of $\Pi$, except the case where $\Pi$ is a polar space of type $D_{n}$ with lines containing precisely three points (Theorem 1). If $k=n-t-1$, where $t$ is an integer satisfying $n \geq 2 t \geq 2$, then $(X, Y) \in \mathfrak{R}_{0, t}$ is equivalent to the fact that $X$ and $Y$ span a maximal singular subspace. Our second result (Theorem 2) states that every bijective transformation of $\mathcal{G}_{k}(\Pi)$ preserving this relation is induced by an automorphism of $\Pi$.

Note that for finite symplectic and hermitian polar spaces, the first result under some conditions was proved in $[18,25]$.

\section{Polar spaces}

We recall some basic properties of polar spaces and refer to [3, 19, 24] for their proofs.

Let $\mathcal{P}$ be a nonempty set whose elements are called points, and $\mathcal{L}$ be a family formed by proper subsets of $\mathcal{P}$ called lines. Two distinct points joined by a line are said to be collinear. Let $\Pi=(P, \mathcal{L})$ be a partial linear space, i.e., each line contains at least two points, and for any distinct collinear points $p, q \in \mathcal{P}$, there is precisely one line containing them, and this line is denoted by $p q$.

We say that $S \subset \mathcal{P}$ is a subspace of $\Pi$ if for any distinct collinear points $p, q \in S$, the line $p q$ is contained in $S$. A singular subspace is a subspace where any two distinct points are collinear. Note that the empty set and a single point are singular subspaces. Using the Zorn lemma, we show that every singular subspace is contained in a certain maximal singular subspace.

From this moment we suppose that $\Pi$ is a polar space. This means that the following axioms hold:

(P1) each line contains at least three points,

(P2) there is no point collinear to all points,

(P3) if $p \in \mathcal{P}$ and $L \in \mathcal{L}$, then $p$ is collinear to precisely one point or all points of the line $L$, 
(P4) every flag formed by singular subspaces is finite.

If there is a maximal singular subspace of $\Pi$ containing more than one line, then all maximal singular subspaces of $\Pi$ are projective spaces of the same finite dimension $\geq 2$. We say that the rank of $\Pi$ is $n$ if this dimension is equal to $n-1$.

In the case where the rank of $\Pi$ is not less than 4, every maximal singular subspace $M$ can be identified with the projective space associated to a certain $n$ dimensional vector space $V$ (over a division ring). Then every nonempty singular subspace $S \subset M$ will be identified with the corresponding subspace of the vector space $V$.

The collinearity relation on $\Pi$ is denoted by $\perp$. We write $p \perp q$ if $p, q \in \mathcal{P}$ are collinear points and $p \not \perp q$ otherwise. If $X, Y \subset \mathcal{P}$, then $X \perp Y$ means that every point of $X$ is collinear to all points of $Y$. For every subset $X \subset \mathcal{P}$ satisfying $X \perp X$, the minimal singular subspace containing $X$ is called spanned by $X$ and denoted by $\langle X\rangle$. For every subset $X \subset \mathcal{P}$, we denote by $X^{\perp}$ the subspace of $\Pi$ formed by all points collinear to all points of $X$.

Fact 1 Let $X$ be a subset of $\mathcal{P}$ satisfying $X \perp X$ and spanning a maximal singular subspace $M$. Then $p \perp X$ implies that $p \in M$.

Fact 2 If $M$ is a maximal singular subspace of $\Pi$, then for every point $p \in \mathcal{P}$ such that $p \notin M$, we have

$$
\operatorname{dim}_{p}\left(p^{\perp} \cap M\right)=n-2 .
$$

Fact 3 For every singular subspace $S$, there are maximal singular subspaces $M$ and $N$ such that $S=M \cap N$.

For every polar space of rank $n$, one of the following possibilities is realized:

- type $\mathrm{C}_{n}$, where every $(n-2)$-dimensional singular subspace is contained in at least three maximal singular subspaces,

- type $\mathrm{D}_{n}$, where every $(n-2)$-dimensional singular subspace is contained in precisely two maximal singular subspaces.

All polar spaces of rank $\geq 3$ were described by Tits [23].

\section{Results}

Let $\Pi=(\mathcal{P}, \mathcal{L})$ be a polar space of rank $n \geq 3$. Recall that the polar Grassmannian formed by all $k$-dimensional singular subspaces of $\Pi$ is denoted by $\mathcal{G}_{k}(\Pi)$. Suppose that $k \leq n-2$. We consider the relation $\mathfrak{R}_{i, j}, 0 \leq i \leq j \leq k+1$, formed by all pairs

$$
(X, Y) \in \mathcal{G}_{k}(\Pi) \times \mathcal{G}_{k}(\Pi)
$$

satisfying

$$
\operatorname{dim}_{p}\left(X^{\perp} \cap Y\right)=k-i \quad \text { and } \quad \operatorname{dim}_{p}(X \cap Y)=k-j .
$$


Since

$$
\operatorname{dim}_{p}\left(X^{\perp} \cap Y\right)=\operatorname{dim}_{p}\left(Y^{\perp} \cap X\right)
$$

for any pair $X, Y \in \mathcal{G}_{k}(\Pi)$, this relation is symmetric. Every automorphism of $\Pi$ (a bijective transformation of $\mathcal{P}$ preserving $\mathcal{L}$ ) induces a transformation of $\mathcal{G}_{k}(\Pi)$ which preserves all $\mathfrak{R}_{i, j}$.

First, we determine all automorphisms of the graph

$$
\Gamma_{k}^{\prime}(\Pi)=\left(\mathcal{G}_{k}(\Pi), \Re_{1,1}\right)
$$

except the case where $\Pi$ is a polar space of type $D_{n}$ with lines containing precisely three points.

Theorem 1 Suppose that one of the following possibilities is realized:

- $\Pi$ is a polar space of type $\mathrm{C}_{n}, n \geq 3$,

- $\Pi$ is a polar space of type $\mathrm{D}_{n}, n \geq 3$, and each line contains more than three points.

Then every automorphism of $\Gamma_{k}^{\prime}(\Pi)$ is induced by an automorphism of $\Pi$.

Remark 1 For $k=0$, this statement is trivial. The edges of $\Gamma_{0}^{\prime}(\Pi)$ are pairs of noncollinear points of $\Pi$, and every automorphism of this graph is an automorphism of the collinearity graph $\Gamma_{0}(\Pi)$. It is well known that the class of automorphisms of $\Gamma_{0}(\Pi)$ coincides with the class of automorphisms of $\Pi$.

In the case where $k \in\{1, \ldots, n-3\}$, the distance between $S, U \in \mathcal{G}_{k}(\Pi)$ in the Grassmann graph $\Gamma_{k}(\Pi)$ is equal to 2 if and only if $(S, U)$ belongs to $\Re_{1,1} \cup \Re_{0,2}$. The distance between $S, U \in \mathcal{G}_{n-2}(\Pi)$ in $\Gamma_{n-2}(\Pi)$ is equal to 2 if and only if $(S, U) \in \mathfrak{R}_{1,1}$ (if $k=n-2$, then $\mathfrak{R}_{0,2}$ is empty). Theorem 1 gives the following.

Corollary 1 Suppose that one of the possibilities from Theorem 1 is realized. Let $f$ be a bijective transformation of $\mathcal{G}_{n-2}(\Pi)$ satisfying the following condition: the distance between $S, U \in \mathcal{G}_{n-2}(\Pi)$ in $\Gamma_{n-2}(\Pi)$ is equal to 2 if and only if the distance between $f(S)$ and $f(U)$ in $\Gamma_{n-2}(\Pi)$ is equal to 2 . Then $f$ is an automorphism of $\Gamma_{n-2}(\Pi)$.

If $X$ and $Y$ are singular subspaces of $\Pi$ contained in a certain maximal singular subspace, then

$$
\operatorname{dim}_{p}(\langle X \cup Y\rangle)=\operatorname{dim}_{p}(X)+\operatorname{dim}_{p}(Y)-\operatorname{dim}_{p}(X \cap Y) .
$$

This implies that a maximal singular subspace of $\Pi$ is spanned by two $k$-dimensional singular subspaces if and only if $n \leq 2 k+2$. In this case, for $X, Y \in \mathcal{G}_{k}(\Pi)$, we write $X \sim Y$ if these singular subspaces span a maximal singular subspace, i.e., $X \perp Y$ and $\langle X \cup Y\rangle$ is a maximal singular subspace. 
Remark 2 Let $X, Y \in \mathcal{G}_{k}(\Pi)$ and

$$
\operatorname{dim}_{p}(X \cap Y)=k-t .
$$

If $X \sim Y$, then (1) implies that

$$
n-1=2 k-(k-t) \text { and } k=n-t-1 .
$$

Since $k+t=n-1$ and $t \leq k+1$, we have $n \geq 2 t$. Therefore, $X \sim Y$ is equivalent to the fact that $k=n-t-1$ and $(X, Y) \in \mathfrak{R}_{0, t}$, where $t$ is an integer satisfying $n \geq 2 t \geq 2$.

Our second result describes all automorphisms of the graph

$$
\Gamma_{k}^{\prime \prime}(\Pi)=\left(\mathcal{G}_{k}(\Pi), \sim\right) .
$$

Recall that this graph is defined only in the case $n \leq 2 k+2$.

Theorem 2 Every automorphism of $\Gamma_{k}^{\prime \prime}(\Pi)$ is induced by an automorphism of $\Pi$.

Remark 3 The graph $\Gamma_{n-2}^{\prime \prime}(\Pi)$ coincides with the Grassmann graph $\Gamma_{n-2}(\Pi)$, and, by [19, Theorem 4.8], every automorphism of this graph is induced by an automorphism of $\Pi$. Thus, we can restrict ourself to the case $k \leq n-3$; in particular, we can suppose that $n \geq 4$.

\section{Cliques}

From this moment we suppose that $k \in\{1, \ldots, n-2\}$. For every singular subspace $N$ such that $\operatorname{dim}_{p} N<k$, we denote by $[N\rangle_{k}$ the set of all elements of $\mathcal{G}_{k}(\Pi)$ containing $N$. This subset is said to be a big star if $N \in \mathcal{G}_{k-1}(\Pi)$.

If $N$ and $M$ are singular subspaces satisfying

$$
N \subset M \text { and } \operatorname{dim}_{p} N<k<\operatorname{dim}_{p} M,
$$

then we denote by $[N, M]_{k}$ the set of all $S \in \mathcal{G}_{k}(\Pi)$ such that $N \subset S \subset M$. This subset is called a star if

$$
N \in \mathcal{G}_{k-1}(\Pi) \quad \text { and } \quad M \in \mathcal{G}_{n-1}(\Pi) .
$$

In the case $N=\emptyset$, we write $\langle M]_{k}$ instead of $[N, M]_{k}$. We say that $\langle M]_{k}$ is a top if $M \in \mathcal{G}_{k+1}(\Pi)$.

All maximal cliques of the Grassmann graph

$$
\Gamma_{k}(\Pi)=\left(\mathcal{G}_{k}(\Pi), \Re_{0,1}\right)
$$

and the weak Grassmann graph

$$
\Gamma_{k}^{w}(\Pi)=\left(\mathcal{G}_{k}(\Pi), \Re_{0,1} \cup \mathfrak{R}_{1,1}\right)
$$


are known [19, Propositions 4.16 and 4.24]. Every maximal clique of $\Gamma_{k}^{w}(\Pi)$ is a big star or a top. Every maximal clique of $\Gamma_{k}(\Pi)$ is a star or a top. In the case $k=n-2$, every star is contained in a certain top, and all maximal cliques of $\Gamma_{k}(\Pi)$ are tops.

Proposition 1 Every clique of $\Gamma_{k}^{\prime}(\Pi)$ is contained in a big star.

Proof If $\mathcal{C}$ is a clique of $\Gamma_{k}^{\prime}(\Pi)$, then it is a clique of $\Gamma_{k}^{w}(\Pi)$. Hence, $\mathcal{C}$ is contained in a big star or a top. Since any two distinct elements of a top are nonadjacent vertices of $\Gamma_{k}^{\prime}(\Pi), \mathcal{C}$ is a subset in a big star.

Let $N \in \mathcal{G}_{k-1}(\Pi)$. For every $M \in \mathcal{G}_{k+1}(\Pi)$ containing $N$, the subset $[N, M]_{k}$ is said to be a line. The big star $[N\rangle_{k}$, together with all lines defined above, is a polar space of rank $n-k$ [19, Lemma 4.4]. This polar space will be denoted by $\Pi_{N}$. If $\Pi$ is a polar space of type $\mathrm{C}_{n}$ or $\mathrm{D}_{n}$, then $\Pi_{N}$ is a polar space of type $\mathrm{C}_{n-k}$ or $\mathrm{D}_{n-k}$, respectively.

Lemma 1 Let $N \in \mathcal{G}_{k-1}(\Pi)$. Two distinct elements of the big star $[N\rangle_{k}$ are adjacent vertices of the graph $\Gamma_{k}^{\prime}(\Pi)$ if and only if they are noncollinear points of the polar space $\Pi_{N}$.

Proof Easy verification.

Proposition 2 Suppose that $n \leq 2 k+2$. If $S$ and $U$ are adjacent vertices of $\Gamma_{k}^{\prime \prime}(\Pi)$, then $M:=\langle S \cup U\rangle$ is a maximal singular subspace of $\Pi$, and every clique of $\Gamma_{k}^{\prime \prime}(\Pi)$ containing $S$ and $U$ is a subset in $\langle M]_{k}$.

Proof It is clear that $S \perp U$ and $M$ is a maximal singular subspace. Let $\mathcal{C}$ be a clique of $\Gamma_{k}^{\prime \prime}(\Pi)$ containing $S$ and $U$. Then for every $A \in \mathcal{C}$, we have $A \perp S$ and $A \perp U$. By Fact 1 , this implies that $A \subset M$. Hence, $\mathcal{C}$ is contained in $\langle M]_{k}$.

\section{Proof of Theorem 1}

Lemma 2 Let $\Pi=(\mathcal{P}, \mathcal{L})$ be a polar space such that one of the following possibilities is realized:

(1) $\Pi$ is a polar space of type $\mathrm{C}_{n}, n \geq 2$;

(2) $\Pi$ is a polar space of type $\mathrm{D}_{n}, n \geq 2$, and each line of $\Pi$ contains more than three points.

Suppose that $p$ and $q$ are noncollinear points of $\Pi$ and $t \in \mathcal{P}$ is collinear to at least one of the points $p, q$. Then there exists a point of $\Pi$ noncollinear to $p, q, t$.

Proof By our assumption, $t$ is collinear to at least one of the points $p$ and $q$. Let $t \perp q$.

(1) Suppose that $\Pi$ is a polar space of type $C_{n}$.

In the case where $t \not \perp p$, we consider a maximal singular subspace $M$ containing the line $q t$. The singular subspace $p^{\perp} \cap M$ is $(n-2)$-dimensional (Fact 2), and there 
is a maximal singular subspace $N$ containing $p^{\perp} \cap M$ and different from $M$ and $\left\langle p^{\perp} \cap M, p\right\rangle$. Every point belonging to $N \backslash\left(p^{\perp} \cap M\right)$ is noncollinear to $p, q, t$.

Now suppose that $t$ is collinear to both $p$ and $q$. Since $p^{\perp} \cap q^{\perp}$ is a polar space of rank $n-1$ (if $n=2$, then $p^{\perp} \cap q^{\perp}$ consists of mutually noncollinear points) [19, Lemma 4.3], there exist a point $u \in p^{\perp} \cap q^{\perp}$ and an $(n-2)$-dimensional singular subspace $U \subset p^{\perp} \cap q^{\perp}$ such that $t \not \perp u$ and $u \in U$. We take a maximal singular subspace $M$ containing $U$ and different from $\langle U, p\rangle$ and $\langle U, q\rangle$. Consider a line $L \subset$ $M$ that intersects $U$ precisely in the point $u$. This line contains a unique point $w$ collinear to $t$. Every point of $L$ different from $u$ and $w$ is noncollinear to $p, q, t$.

(2) Consider the case where each line of $\Pi$ contains more than three points (we do not assume that the polar space is of type $D_{n}$ ). Suppose that the statement fails and every point of $\Pi$ is collinear to at least one of the points $p, q, t$ or, in other words,

$$
\mathcal{P}=p^{\perp} \cup q^{\perp} \cup t^{\perp} .
$$

First we show that every maximal singular subspace of $\Pi$ contains at least one of the points $p, q, t$.

Let $M$ be a maximal singular subspace of $\Pi$. If each of the points $p, q, t$ does not belong to $M$, then

$$
p^{\perp} \cap M, q^{\perp} \cap M, t^{\perp} \cap M
$$

are $(n-2)$-dimensional subspaces of $M$ (Fact 2), and (2) implies that $M$ is the union of these subspaces. The latter is impossible since a projective space cannot be presented as the union of three hyperplanes if each line contains more than three points.

The line $q t$ contains the unique point $s$ collinear to $p$. One of the following possibilities is realized:

(1) $s=t$,

(2) $s \neq t$.

In the case (1), we take any point $v$ on the line $q t$ different from $q$ and $t$. It is clear that $p \not \perp v$. By Fact 3, there exist maximal singular subspaces $M$ and $N$ such that $M \cap$ $N=\{v\}$. Since $p \not \perp v$, they do not contain $p$. Then one of these subspaces contains $q$, and the other contains $t$. So, each of these subspaces contains two distinct points of the line $q t$. This means that this line is contained in $M \cap N$, which is impossible.

In the case (2), we take any point $w$ on the line $p s$ different from $p$ and $s$. As in the previous case, we consider maximal singular subspaces $M$ and $N$ such that $M \cap N=\{w\}$. One of these subspaces contains $q$ or $t$. Then at least one of the points $q, t$ is collinear to $w$. Since $q$ and $t$ both are collinear to $s$ and $w$ is on the line $p s$, one of the points $q, t$ is collinear to all points of the line $p s$. Thus, $p$ is collinear to $q$ or $t$, which is impossible.

Remark 4 If $\Pi$ is a polar space of type $D_{2}$ and each line contains precisely three points, then $\Pi$ is a grid consisting of nine points. There are points $p, q, t$ such that $p \not \perp q$ and $q \perp t$, and every point of $\Pi$ is collinear to at least one of the points $p, q, t$. See Fig. 1.

From this moment we suppose that $\Pi=(\mathcal{P}, \mathcal{L})$ is a polar space of rank $n \geq 3$ satisfying one of the conditions from Lemma 2. Let also $k \in\{1, \ldots, n-2\}$. 
Fig. 1 A grid

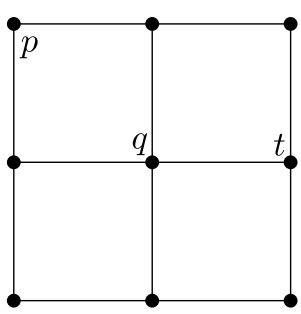

Lemma 3 Suppose that $N \in \mathcal{G}_{k-1}(\Pi)$. Let $P, Q \in[N\rangle_{k}$ be adjacent vertices of $\Gamma_{k}^{\prime}(\Pi)$, and let $T \in[N\rangle_{k}$ be a vertex of $\Gamma_{k}^{\prime}(\Pi)$ nonadjacent to at least one of the vertices $P, Q$. Then there exists $S \in[N\rangle_{k}$ adjacent to $P, Q, T$ in $\Gamma_{k}^{\prime}(\Pi)$.

Proof By Lemma 1, $P$ and $Q$ are noncollinear points of $\Pi_{N}$, and $T$ is a point of $\Pi_{N}$ collinear to at least one of the points $P, Q$. We apply Lemma 2 to the polar space $\Pi_{N}$ ( $\Pi$ satisfies the conditions of Lemma 2 , and the same holds for $\left.\Pi_{N}\right)$ and get the claim.

Remark 5 Let $k=n-2$. Then $\Pi_{N}$ is a polar space of rank 2. If $\Pi$ is a polar space of type $\mathrm{D}_{n}$ and each line contains precisely three points, then $\Pi_{N}$ is a polar space of type $\mathrm{D}_{2}$ with lines containing precisely three points, and Remark 4 shows that Lemma 3 fails.

Let $f$ be an automorphism of $\Gamma_{k}^{\prime}(\Pi)$.

Let us show that $f$ transfers big stars to subsets of big stars. We take any $N \in$ $\mathcal{G}_{k-1}(\Pi)$. Let $P$ and $Q$ be adjacent vertices of $\Gamma_{k}^{\prime}(\Pi)$ contained in the big $\operatorname{star}[N\rangle_{k}$. Then $f(P)$ and $f(Q)$ are adjacent vertices of $\Gamma_{k}^{\prime}(\Pi)$ contained in the big star $\left[N^{\prime}\right\rangle_{k}$, where

$$
N^{\prime}=f(P) \cap f(Q) .
$$

We assert that $f(T) \in\left[N^{\prime}\right\rangle_{k}$ for every $T \in[N\rangle_{k}$ and prove this statement in several steps.

(i) First, we consider the case where $T$ is a vertex of $\Gamma_{k}^{\prime}(\Pi)$ adjacent to both $P$ and $Q$. Then $f(P), f(Q), f(T)$ form a clique in $\Gamma_{k}^{\prime}(\Pi)$ that, by Proposition 1, is contained in a certain big star $\left[N^{\prime \prime}\right\rangle_{k}$. We have

$$
N^{\prime}=f(P) \cap f(Q)=N^{\prime \prime},
$$

which gives the claim.

(ii) Consider the case where $T$ is a vertex of $\Gamma_{k}^{\prime}(\Pi)$ adjacent to precisely one of the vertices $P, Q$. Suppose that $T$ is adjacent to $P$. Lemma 3 implies the existence of a vertex $S \in[N\rangle_{k}$ in the graph $\Gamma_{k}^{\prime}(\Pi)$ adjacent to $P, Q, T$. By (i), $f(S)$ belongs to $\left[N^{\prime}\right\rangle_{k}$. Then

$$
f(P) \cap f(S)=N^{\prime},
$$

and $f(P), f(S), f(T)$ form a clique of $\Gamma_{k}^{\prime}(\Pi)$. As in (i), we show that $f(T) \in\left[N^{\prime}\right\rangle_{k}$. 
(iii) Suppose that $T$ is a vertex of $\Gamma_{k}^{\prime}(\Pi)$ nonadjacent to both $P$ and $Q$. As above, we consider $S \in[N\rangle_{k}$ that is a vertex of $\Gamma_{k}^{\prime}(\Pi)$ adjacent to $P, Q, T$ and obtain that $f(S) \in\left[N^{\prime}\right\rangle_{k}$. We apply the arguments from (ii) to $P, S, T$ and establish that $f(T) \in$ $\left[N^{\prime}\right\rangle_{k}$

So, $f$ transfers big stars to subsets of big stars. The same arguments show that $f^{-1}$ sends big stars to subsets of big stars. This means that $f$ and $f^{-1}$ both map big stars to big stars, i.e., there exists a bijective transformation $g$ of $\mathcal{G}_{k-1}(\Pi)$ such that

$$
f\left([N\rangle_{k}\right)=[g(N)\rangle_{k}
$$

for every $N \in \mathcal{G}_{k-1}(\Pi)$.

Let $U \in \mathcal{G}_{k}(\Pi)$. Then $g$ transfers the top $\langle U]_{k-1}$ to the top $\langle f(U)]_{k-1}$. Indeed, we have

$$
N \in\langle U]_{k-1} \quad \Leftrightarrow \quad U \in[N\rangle_{k} \Leftrightarrow f(U) \in[g(N)\rangle_{k} \Leftrightarrow g(N) \in\langle f(U)]_{k-1} .
$$

Similarly, $g^{-1}$ sends $\langle U]_{k-1}$ to the top $\left\langle f^{-1}(U)\right]_{k-1}$.

Therefore, $g$ and $g^{-1}$ both transfer tops to tops. Since for any two adjacent vertices of the Grassmann graph $\Gamma_{k-1}(\Pi)$, there is a top containing them, $g$ is an automorphism of $\Gamma_{k-1}(\Pi)$.

By [19, Theorems 4.8 and 4.9], every automorphism of $\Gamma_{k-1}(\Pi)$ is induced by an automorphism of $\Pi$ except the case where $k=2$ and $\Pi$ is a polar space of type $D_{4}$. In this special case, every automorphism of $\Gamma_{k-1}(\Pi)$ is induced by an automorphism of $\Pi$ or an isomorphism of $\Pi$ to one of the half-spin Grassmann spaces of $\Pi[19$, Theorem 4.9]. Automorphisms of the second type (induced by isomorphisms of $\Pi$ to the half-spin Grassmann spaces) do not send tops to tops. This means that $g$ is an automorphism of the first type.

So, $g$ is induced by an automorphism of $\Pi$. An easy verification shows that this automorphism also induces $f$.

\section{Proof of Theorem 2}

By Remarks 2 and 3, we can suppose that $n \geq 4$ and $k=n-t-1$, where $t$ is an integer satisfying $n \geq 2 t \geq 4$, and for every $X, Y \in \mathcal{G}_{k}(\Pi)$, we have

$$
X \sim Y \quad \operatorname{dim}_{p}\left(X^{\perp} \cap Y\right)=k, \quad \operatorname{dim}_{p}(X \cap Y)=k-t .
$$

It is well known that every polar space of rank $\geq 4$ is embeddable in a projective space. This means that every maximal singular subspace of $\Pi$ can be considered as the projective space associated to an $n$-dimensional vector space.

Lemma 4 Let $S, U$ be adjacent vertices of $\Gamma_{k}^{\prime \prime}(\Pi)$. Let also $M$ be the maximal singular subspace spanned by $S$ and $U$. If $T \in\langle M]_{k}$ is a vertex of $\Gamma_{k}^{\prime \prime}(\Pi)$ nonadjacent to at least one of the vertices $S, U$, then there exists a vertex $Q$ of $\Gamma_{k}^{\prime \prime}(\Pi)$ adjacent to $S, U, T$. 
Proof We suppose that $T$ is nonadjacent to $S$ (the case where $T$ is nonadjacent to $U$ is similar). First of all, we show that the general case can be reduced to the case where $N:=S \cap U$ and $T$ are disjoint.

Suppose that the projective dimension of $W:=N \cap T$ is equal to $w \geq 0$. Then $\Pi_{W}$ is a polar space of rank $n-w-1$, and every $k$-dimensional singular subspace of $\Pi$ containing $W$ can be considered as a $(k-w-1)$-dimensional singular subspace of $\Pi_{W}$. Two $k$-dimensional singular subspaces containing $W$ span a maximal singular subspace of $\Pi$ if and only if the corresponding $(k-w-1)$-dimensional singular subspaces span a maximal singular subspace of $\Pi_{W}$. Therefore, the graph $\Gamma_{k-w-1}^{\prime \prime}\left(\Pi_{W}\right)$ is naturally isomorphic to the subgraph of $\Gamma_{k}^{\prime \prime}(\Pi)$ induced on $[W\rangle_{k}$, and $S, U, T$ can be considered as vertices of $\Gamma_{k-w-1}^{\prime \prime}\left(\Pi_{W}\right)$.

So, we restrict ourself to the case where $N$ and $T$ are disjoint. We identify $M$ with the projective space associated to a certain $n$-dimensional vector space $V$. Every nonempty singular subspace of $M$ will be identified with the corresponding subspace of the vector space $V$. We set

$$
m:=\operatorname{dim}_{l} N=n-2 t
$$

and suppose that

$$
\operatorname{dim}_{l}(S \cap T)=i, \quad \operatorname{dim}_{l}(U \cap T)=j .
$$

Note that $i>m$ since $S$ and $T$ are nonadjacent vertices of $\Gamma_{k}^{\prime \prime}(\Pi)$.

First, we consider the case where $i+j=n-t$, i.e., $T$ is spanned by $S \cap T$ and $U \cap T$. Since $\operatorname{dim}_{l}(S / N)=\operatorname{dim}_{l}(U / N)=t$, we can choose vectors

$$
x_{1}, \ldots, x_{t} \in S \backslash N \quad \text { and } \quad y_{1}, \ldots, y_{t} \in U \backslash N
$$

such that

$$
\begin{aligned}
& S=N+\left\langle x_{1}, \ldots, x_{t}\right\rangle, \quad U=N+\left\langle y_{1}, \ldots, y_{t}\right\rangle, \\
& T=\left\langle x_{1}, \ldots, x_{i}\right\rangle+\left\langle y_{t-j+1}, \ldots, y_{t}\right\rangle .
\end{aligned}
$$

The vectors $x_{1}+y_{1}, \ldots, x_{t}+y_{t}$ are linearly independent. We define

$$
Q:=N+\left\langle x_{1}+y_{1}, \ldots, x_{t}+y_{t}\right\rangle .
$$

An easy verification shows that

$$
S+Q=U+Q=T+Q=M,
$$

which implies that $Q$ is a vertex of $\Gamma_{k}^{\prime \prime}(\Pi)$ adjacent to $S, U, T$.

Now suppose that

$$
l:=n-t-(i+j)>0 .
$$

Then $t=i+j-m+l$. Since $i>m$, we have $i+j-m>0$. We choose linearly independent vectors

$$
x_{1}, \ldots, x_{i+j-m}, x_{1}^{\prime}, \ldots, x_{l}^{\prime} \in S \backslash N
$$


and linearly independent vectors

$$
y_{1}, \ldots, y_{i+j-m}, y_{1}^{\prime}, \ldots, y_{l}^{\prime} \in U \backslash N
$$

such that

$$
T=\left\langle x_{1}, \ldots, x_{i}\right\rangle+\left\langle y_{i-m+1}, \ldots, y_{i+j-m}\right\rangle+\left\langle x_{1}^{\prime}+y_{1}^{\prime}, \ldots, x_{l}^{\prime}+y_{l}^{\prime}\right\rangle .
$$

We denote by $Q$ the subspace spanned by $N$ and the vectors

$$
x_{1}+y_{1}^{\prime}, x_{1}^{\prime}+y_{2}^{\prime}, \ldots, x_{l-1}^{\prime}+y_{l}^{\prime}, x_{l}^{\prime}+y_{1}, x_{2}+y_{2}, \ldots, x_{i+j-m}+y_{i+j-m} .
$$

Using the equalities

$$
\begin{aligned}
& S=N+\left\langle x_{1}, \ldots, x_{i+j-m}, x_{1}^{\prime}, \ldots, x_{l}^{\prime}\right\rangle, \\
& U=N+\left\langle y_{1}, \ldots, y_{i+j-m}, y_{1}^{\prime}, \ldots, y_{l}^{\prime}\right\rangle,
\end{aligned}
$$

we establish that $S+Q=U+Q=M$. To complete the proof, we need to check that $T+Q=M$.

The conditions

$$
x_{1}, \ldots, x_{i}, y_{i-m+1}, \ldots, y_{i+j-m} \in T \quad \text { and } \quad x_{2}+y_{2}, \ldots, x_{i+j-m}+y_{i+j-m} \in Q
$$

imply that $x_{p} \in T+Q$ for every $p$ and $y_{p} \in T+Q$ if $p \geq 2$. Since $x_{1} \in T$ and $x_{1}+y_{1}^{\prime} \in Q$, we have $y_{1}^{\prime} \in T+Q$. Then $x_{1}^{\prime}+y_{1}^{\prime} \in T$ implies that $x_{1}^{\prime} \in T+Q$. Step by step, we establish that all $x_{q}^{\prime}$ and $y_{q}^{\prime}$ belong to $T+Q$. The conditions $x_{l}^{\prime} \in$ $T+Q$ and $x_{l}^{\prime}+y_{1} \in Q$ guarantee that $y_{1} \in T+Q$. Therefore, $Q+T$ coincides with $S+U=M$.

Let $f$ be an automorphism of $\Gamma_{k}^{\prime \prime}(\Pi)$. We show that for every $M \in \mathcal{G}_{n-1}(\Pi)$, there exists $M^{\prime} \in \mathcal{G}_{n-1}(\Pi)$ such that

$$
f\left(\langle M]_{k}\right) \subset\left\langle M^{\prime}\right]_{k} .
$$

We choose $S, U \in\langle M]_{k}$ such that $M$ is spanned by $S \cup U$. Then $S$ and $U$ are adjacent vertices of $\Gamma_{k}^{\prime \prime}(\Pi)$, and the same holds for $f(S)$ and $f(U)$. Hence,

$$
M^{\prime}:=\langle f(S) \cup f(U)\rangle
$$

is a maximal singular subspace. We assert that $f(T) \in\left\langle M^{\prime}\right]_{k}$ for every $T \in\langle M]_{k}$ and prove this statement in several steps.

(i) If $T \in\langle M]_{k}$ is a vertex of $\Gamma_{k}^{\prime \prime}(\Pi)$ adjacent to $S$ and $U$, then $f(S), f(U), f(T)$ form a clique in $\Gamma_{k}^{\prime \prime}(\Pi)$, and, by Proposition 2, we have $f(T) \in\left\langle M^{\prime}\right]_{k}$.

(ii) Now, let $T \in\langle M]_{k}$ be a vertex of $\Gamma_{k}^{\prime \prime}(\Pi)$ adjacent to precisely one of the vertices $U$ and $S$. Suppose that $T$ is adjacent to $U$ and nonadjacent to $S$. Let $Q \in$ $\langle M]_{k}$ be a vertex of $\Gamma_{k}^{\prime \prime}(\Pi)$ adjacent to $S, U, T$ (Lemma 4). By (i), $f(Q)$ belongs to $\left\langle M^{\prime}\right]_{k}$. Then

$$
\langle f(U) \cup f(Q)\rangle=M^{\prime}
$$


We apply (i) to $U, Q, T$ and establish that $f(T)$ belongs to $\left\langle M^{\prime}\right]_{k}$.

(iii) Consider the case where $T$ is a vertex of $\Gamma_{k}^{\prime \prime}(\Pi)$ nonadjacent to both $S$ and $U$. As above, we consider $Q \in\langle M]_{k}$ that is a vertex of $\Gamma_{k}^{\prime \prime}(\Pi)$ adjacent to $S, U, T$. Then $f(Q) \in\left\langle M^{\prime}\right]_{k}$, and (3) holds. We apply (ii) to $U, Q, T$ and obtain that $f(T) \in\left\langle M^{\prime}\right]_{k}$.

We apply the above arguments to $f^{-1}$ and establish the existence of a bijective transformation $g$ of $\mathcal{G}_{n-1}(\Pi)$ such that

$$
f\left(\langle M]_{k}\right)=\langle g(M)]_{k}
$$

for every $M \in \mathcal{G}_{n-1}(\Pi)$.

Let $U$ be a singular subspace of $\Pi$ such that $k<\operatorname{dim}_{p} U<n-1$. Then there exist $M_{1}, M_{2} \in \mathcal{G}_{n-1}(\Pi)$ satisfying $U=M_{1} \cap M_{2}$. We have

$$
\begin{aligned}
\langle U]_{k} & =\left\langle M_{1}\right]_{k} \cap\left\langle M_{2}\right]_{k}, \\
f\left(\langle U]_{k}\right) & =\left\langle g\left(M_{1}\right)\right]_{k} \cap\left\langle g\left(M_{2}\right)\right]_{k}=\left\langle g\left(M_{1}\right) \cap g\left(M_{2}\right)\right]_{k} .
\end{aligned}
$$

We set

$$
g(U):=g\left(M_{1}\right) \cap g\left(M_{2}\right)
$$

and get an extension of $g$ to a transformation of

$$
\mathcal{G}_{k+1}(\Pi) \cup \cdots \cup \mathcal{G}_{n-1}(\Pi)
$$

such that

$$
f\left(\langle U]_{k}\right)=\langle g(U)]_{k}
$$

for every $U$ belonging to (4). We apply the same arguments to the pair $g^{-1}, f^{-1}$ and show that $g$ is bijective. It is clear that $g$ is inclusion preserving, i.e.,

$$
S \subset U \Longleftrightarrow g(S) \subset g(U)
$$

for any $S, U$ belonging to (4). The latter guarantees that $g$ sends $\mathcal{G}_{i}(\Pi)$ to itself for every integer $i$ satisfying $k<i \leq n-1$. Therefore, $f$ and $f^{-1}$ both send tops to tops, which implies that $f$ is induced by an automorphism of $\Pi$.

Acknowledgements The authors are grateful to the referee for remarks concerning Lemma 2 and Theorem 2. W. Liu was supported by NSFC (11301138, 11271004). K. Wang was supported by NSFC (11271047, 11371204) and the Fundamental Research Funds for the Central University of China.

\section{References}

1. Abramenko, P., Van Maldeghem, H.: On opposition in spherical buildings and twin buildings. Ann. Comb. 4, 125-137 (2000)

2. Blunck, A., Havlicek, H.: On bijections that preserve complementarity of subspaces. Discrete Math. 301, 46-56 (2005)

3. Buekenhout, F., Cohen, A.M.: Diagram Geometry. Springer, Berlin (2013)

4. Chow, W.L.: On the geometry of algebraic homogeneous spaces. Ann. Math. 50, 32-67 (1949) 
5. Govaert, E., Van Maldeghem, H.: Distance-preserving maps in generalized polygons. Part I: maps on flags. Beitr. Algebra Geom. 43, 89-110 (2002)

6. Govaert, E., Van Maldeghem, H.: Distance-preserving maps in generalized polygons. Part II: maps on points and/or lines. Beitr. Algebra Geom. 43, 303-324 (2002)

7. Havlicek, H.: On isomorphisms of Grassmann spaces. Mitt. Math. Ges. Hamb. 14, 117-120 (1995)

8. Havlicek, H.: Chow's theorem for linear spaces. Discrete Math. 208/209, 319-324 (1999)

9. Havlicek, H., Pankov, M.: Transformations on the product of Grassmann spaces. Demonstr. Math. 38, 675-688 (2005)

10. Huang, W.-1.: Adjacency preserving transformations of Grassmann spaces. Abh. Math. Semin. Univ. Hamb. 68, 65-77 (1998)

11. Huang, W.-1.: Adjacency preserving mappings of invariant subspaces of a null system. Proc. Am. Math. Soc. 128, 2451-2455 (2000)

12. Huang, W.-1.: Characterization of the transformation group of the space of a null system. Results Math. 40, 226-232 (2001)

13. Huang, W.-1.: Bounded distance preserving surjections in the geometry of matrices. Linear Algebra Appl. 433, 1973-1987 (2010)

14. Huang, W.-1.: Bounded distance preserving surjections in the projective geometry of matrices. Linear Algebra Appl. 435, 175-185 (2011)

15. Huang, W.-1., Havlicek, H.: Diameter preserving surjections in the geometry of matrices. Linear Algebra Appl. 429, 376-386 (2008)

16. Kreuzer, A.: On isomorphisms of Grassmann spaces. Aequ. Math. 56, 243-250 (1998)

17. Lim, M.H.: Surjections on Grassmannians preserving pairs of elements with bounded distance. Linear Algebra Appl. 432, 1703-1707 (2010)

18. Liu, W., Ma, C., Wang, K.: Full automorphism group of generalized unitary graphs. Linear Algebra Appl. 437, 684-691 (2012)

19. Pankov, M.: Grassmannians of Classical Buildings. Algebra and Discrete Math. vol. 2. World Scientific, Singapore (2010)

20. Pankov, M.: Metric characterization of apartments in dual polar spaces. J. Comb. Theory, Ser. A 118, 1313-1321 (2011)

21. Pankov, M.: Embeddings of Grassmann graphs. Linear Algebra Appl. 436, 3413-3424 (2012)

22. Pankov, M., Prażmovski, K., Żynel, M.: Geometry of polar Grassmann spaces. Demonstr. Math. 39, 625-637 (2006)

23. Tits, J.: Buildings of Spherical Type and Finite BN-Pairs. Lecture Notes in Mathematics, vol. 386. Springer, Berlin (1974)

24. Ueberberg, J.: Foundations of Incidence Geometry. Projective and Polar Spaces. Springer, Berlin (2011)

25. Zeng, L., Chai, Z., Feng, R., Ma, C.: Full automorphism group of the generalized symplectic graph. Sci. China Math. 56, 1509-1520 (2013) 